\title{
Identifying optimal therapies in patients with advanced hepatocellular carcinoma: a systematic review and network meta-analysis
}

\author{
Zhenyu Yang ${ }^{1 \#}$, Yao Tong ${ }^{2 \#}$, Lin Yang ${ }^{1 \#}$, Xianli He ${ }^{1}$, Guoqiang Bao ${ }^{1}$, Xilin Du ${ }^{1}$ \\ ${ }^{1}$ Department of General Surgery, Tangdu Hospital, Air Force Military Medical University, Xi'an, China; ${ }^{2}$ Department of Anesthesiology, Tangdu \\ Hospital, Air Force Military Medical University, Xi'an, China \\ Contributions: (I) Conception and design: Z Yang, Y Tong; (II) Administrative support: X He; (III) Provision of study materials or patients: L Yang, \\ X He, G Bao, X Du; (IV) Collection and assembly of data: Z Yang, Y Tong; (V) Data analysis and interpretation: Z Yang, L Yang; (VI) Manuscript \\ writing: All authors; (VII) Final approval of manuscript: All authors. \\ \#These authors contributed equally to this work. \\ Correspondence to: Xilin Du; Guoqiang Bao. Department of General Surgery, Tangdu Hospital, Air Force Military Medical University, Xi'an 710038, \\ China. Email: dux10705@fmmu.edu.cn; guoqiang@fmmu.edu.cn.
}

Background: Recently, increasing literature has been reported on optimal therapies in patients with advanced hepatocellular carcinoma (HCC) and many therapeutic modalities have been proposed to improve the survival rate. However, the results are not consistent due to different research protocols, small sample sizes and different study endpoints and there is no standard treatment protocol has been defined. Therefore, it is very important to explore the optimal bonding mode and to evaluate the efficacy and safety of the optimal sequential therapy for those patients.

Methods: We searched available databases through January 2020 for relevant studies. The main outcome measure was 1-year overall survival (OS) and overall response rate (ORR); the secondary outcome measure was a composite of toxic effects retrieved grade 3 or 4 adverse events (AEs) from all included studies. Statistical analyses were conducted using STATA version 15 and GeMTC package in the R statistical software.

Results: After a detailed review, 8 randomized controlled trials (RCTs) and 20 retrospective studies involving 3,675 advanced HCC patients were included for network meta-analysis. Indirect comparisons showed that hepatic arterial infusion chemotherapy (HAIC) plus radiofrequency ablation (RFA) was highest probability of obtaining the best OS rate of 1 year [surface under the cumulative ranking (SUCRA), 0.95] and ORR (SUCRA, 0.86) when compared with other potential optimal therapies and which had ranked the first in all treatment regimens, followed by HAIC (SUCRA, 0.75). Direct and indirect comparison of 1 -year OS and ORR with all treatment regimens each other showed that for all treatment regimens, patients showed significant clinical benefit when compared with transcatheter arterial chemoembolization (TACE) or sorafenib alone. However, the incidence of treatment-related AEs of grade 3 or 4 occurred in patients who have received targeted drug sorafenib therapy (SUCRA, 0.51) compared with other interesting regimens.

Conclusions: HAIC may be a valuable therapeutic strategy for advanced HCC patients to prevent recurrence and metastasis after RFA, as well as in improving patient prognosis and quality of life. Meanwhile, HAIC combined with RFA is a safe and effective treatment in patients with advanced HCC, and this combination therapy can significantly prolong 1-year survival rate when compared with other optimal sequential therapies.

Trial registration: This study is registered with PROSPERO, number CRD42020176149.

Keywords: Advanced hepatocellular carcinoma (advanced HCC); network meta-analysis; optimal sequential therapy; survival analysis

Received: 14 October 2020; Accepted: 28 January 2021; Published: 25 October 2022.

doi: $10.21037 /$ tgh-20-318

View this article at: http://dx.doi.org/10.21037/tgh-20-318 


\section{Introduction}

Hepatocellular carcinoma (HCC) is the most prevalent malignant tumor worldwide and the third leading source of cancer mortality with about 750,000 people dying of HCC each year. Hepatitis B virus (HBV), especially in China, is one of the main causes of HCC (1). The occurrence and development of HCC is a multi-factor, multi-step complex process, which is affected by dual factors of environment and diet, which is associated with HBV and hepatitis C virus (HCV) infection, alcohol, cirrhosis, sex hormones, nitrosamines material related (2). With the advent of new $\mathrm{HCV}$ therapies, HCV-related HCC may decrease, and nonalcoholic steatohepatitis-related HCC caused by diabetes and obesity may increase with the increasing prevalence of related diseases, and become the main cause of HCC.

The prognosis of HCC depends primarily on the stage of the tumor at diagnosis. The 5 -year survival rate is more than $70 \%$ for early tumors and only $1-2$ years for advanced tumors. The current treatment options for HCC mainly include surgical resection, transplantation, interventional chemoembolization, radiofrequency ablation (RFA), radiotherapy (RT) and targeted drug therapy (3). With the advancements in therapeutic modalities, the first-line and second-line treatment for advanced HCC has undergone a tremendous transformation, the survival rate of HCC patients also has been significantly improved $(4,5)$. Patients could benefit from many treatment options such as the tyrosine-kinase inhibitor (TKI) (6), cellular immunotherapy (CIT) and transcatheter arterial chemoembolization (TACE) or hepatic arterial infusion chemotherapy (HAIC) predominant local minimally-invasive treatment (7).

TACE, RFA as the most commonly used minimally invasive treatments, have also been used to reduce tumor size and increase tumor necrosis, which also have been widely used to prolong survival time for patients with advanced HCC in combination with systemic therapeutics such as targeted or CIT drugs. Through several studies and meta-analysis had revealed that the combination of TACE and sorafenib was more effective, another randomized controlled trials (RCTs) study have shown the opposite result. And with a lack of a direct head-to-head comparison between many single system therapies or/and compared with local treatment regimens for advanced HCC patients, the choice of optimal therapies regimens for advanced HCC patients has not reached a consensus until now.

Therefore, this network meta-analysis aimed to analyses relevant clinical trials of systemic therapeutics (including
TKI and CIT) combined with optimal minimally invasive treatment in recent years as much as possible to evaluate the efficacy of systemic therapeutics used for advanced HCC patients and ascertain the benefits of combination therapy. We present the following article in accordance with the PRISMA reporting checklist (available at https://tgh. amegroups.com/article/view/10.21037/tgh-20-318/rc).

\section{Methods}

\section{Search strategy for identification of studies}

The following search terms were used to identify comparative studies in PubMed, Web of Science and the Cochrane Library published from January 2010 to January 2020: [advanced hepatocellular carcinoma OR advanced HCC] AND [sorafenib OR tyrosine-kinase inhibitor (6)] OR [transcatheter arterial chemoembolization OR TACE OR hepatic arterial infusion chemotherapy OR HAIC OR chemoembolization] OR [radiofrequency ablation OR RFA OR ablation] OR [radiotherapy] OR [immunotherapy OR cellular immunotherapy OR immune checkpoint inhibitors]. These terms were used in different combinations. The references of retrieved articles were also screened. Each titles or abstracts was evaluated by a single investigator, but was reviewed by two investigators ( $\mathrm{ZY}$ and $\mathrm{LY}$ ) independently for final inclusion and data extraction. The search was limited to English articles involving only adult patients.

\section{Study selection and inclusion criteria}

Only phase II and III RCTs and retrospective propensity score matching studies that compared different interventional therapies for advanced HCC were eligible, including a systemic therapeutic (including TKI and CIT) drug comparing, or comparing a systemic therapeutic drug with conventional therapy or comparing two different local minimally-invasive treatment. Only advanced HCC diagnosed by computed tomography (CT) or magnetic resonance imaging (MRI) according to Barcelona Clinic Liver Cancer (BCLC) staging system were accepted. Necessary information included overall survival (OS), overall response rate (ORR) and adverse events (AEs). The study with multiple arms was preferred as much as possible so as to build comparative loops in network meta-analysis.

\section{Exclusion criteria}

We excluded conference abstracts, letters, case reports, 
reviews, meta-analyses, without control or placebo-controlled studies and single-arm RCTs, studies that only compared efficacy of combination therapy versus a systemic therapeutic alone were excluded. In addition, we also excluded some presentations of ongoing RCT studies because these brief reports lacked useful survival information or did not contain detailed safety data. Notably, according to study objects, the major reason for exclusion of studies including HCC patients with BCLC stage B or A and hepatic metastases or mixed malignancies.

\section{Data extraction and quality assessment}

ZY and LY independently screened and extracted following baseline characteristics data from included studies using a predefined template: included basic information (first author, date of publication, country and study design), participants information and diseases characteristics, intervention methods (such as total number of patients, arms and treatment regimens, interventions, Child-Pugh stage, length of follow up) and outcomes (OS, ORR and major complications or grade 3 or $4 \mathrm{AE}$ ).

The risk of data bias for the original study assessed for quality by another author (YT) or by discussion. To assess the risk of bias in the observational studies, we utilized the Newcastle-Ottawa Quality Assessment Scale (NOS) and major prognostic factors of HCC, which included patient selection, comparability of different interventional therapies for advanced HCC, and assessment of outcome. The risk of bias in RCTs was assessed based on Cochrane Handbook for Systematic Reviews of Interventions recommendations. Each bias item was assessed as high risk, low risk or unclear. And disagreements were resolved through discussion.

\section{Statistical analysis}

Traditional pairwise meta-analyses were conducted using a random-effects model. Begg's and Egger's tests were used to detect publication bias. Bayesian network meta-analysis was carried out to simultaneously compare all interventions in the network. Data of OS, ORR and treatment-related AEs were extracted from included studies and evaluated in both traditional pairwise meta-analyses and Bayesian network meta-analysis which were reported as hazard ratios (HRs) and its $95 \%$ confidence intervals (8). Convergence was assessed to calculate the potential scale reduction factor (PSRF), and values were limited to 1 to complete the calculation which were confirmed after 4 chains, a 50,000-simulation burn- in phase and a thinning interval of 10 for each chain. The values of the surface under the cumulative ranking (SUCRA) probabilities are presented to clarify the pros and cons of different treatment modalities. Different ranks of included treatment strategies are presented based on the SUCRA values, and the optimal method would be determined with the highest SUCRA. Additionally, a node-splitting analysis was applied to check the consistency between direct evidence and indirect evidence for their agreement on a specific node. Statistical analyses were conducted using STATA version 15 and GeMTC package in the R statistical software (version 3.6.1, R Foundation).

\section{Results}

\section{Study characteristics and quality assessment}

The participant flow chart of this study selection procedure, according to the PRISMA statement, is depicted in Figure 1. The Literature searches yielded 1,918 potentially relevant references. According to title and abstract, 1,357 publications were excluded because they did not meet the research criteria. A further 41 were excluded after detailed assessment of the full text. Finally, A total of 28 studies enrolling 3,675 advanced HCC participants who received at least two different optimal therapies were included in our network meta-analysis, which were all published in English (Table 1). The detailed characteristics of included relevant studies for this network meta-analysis were described in Table 1. which contained 8 RCTs $(6,9-23)$ and 20 retrospective studies $(10,24-41)$.

Figure 2 shows that 28 relevant studies evaluating 10 different treatment methods (TACE, sorafenib, HAIC, TACE + sorafenib, TACE + RT, RFA + sorafenib, HAIC + sorafenib, HAIC + RT, HAIC + RFA, TACE + RT + sorafenib) were included in the optimal therapies-based network meta-analysis. The study sample sizes of patients ranged from 16 to 189 and the duration of follow-up ranged from 24 to 60 months. All the 3,675 participants conformed to the inclusion and exclusion criteria and had well compensated liver reserve function with Child-Pugh A or B. Meanwhile, most of patients ECOG PS was reported to be 0 or 1 . The regimens of HAIC was conventional used include cisplatin (CDDP) only, CDDP plus 5-fluorouracil (5-FU), and TACE was in conventional interventions in 10 studies and used drug-eluting beads (DEB) in 4 studies. The median number of HAIC and TACE sessions ranged from 6 to 12 and 2 to 4 , respectively. We mainly compared the 


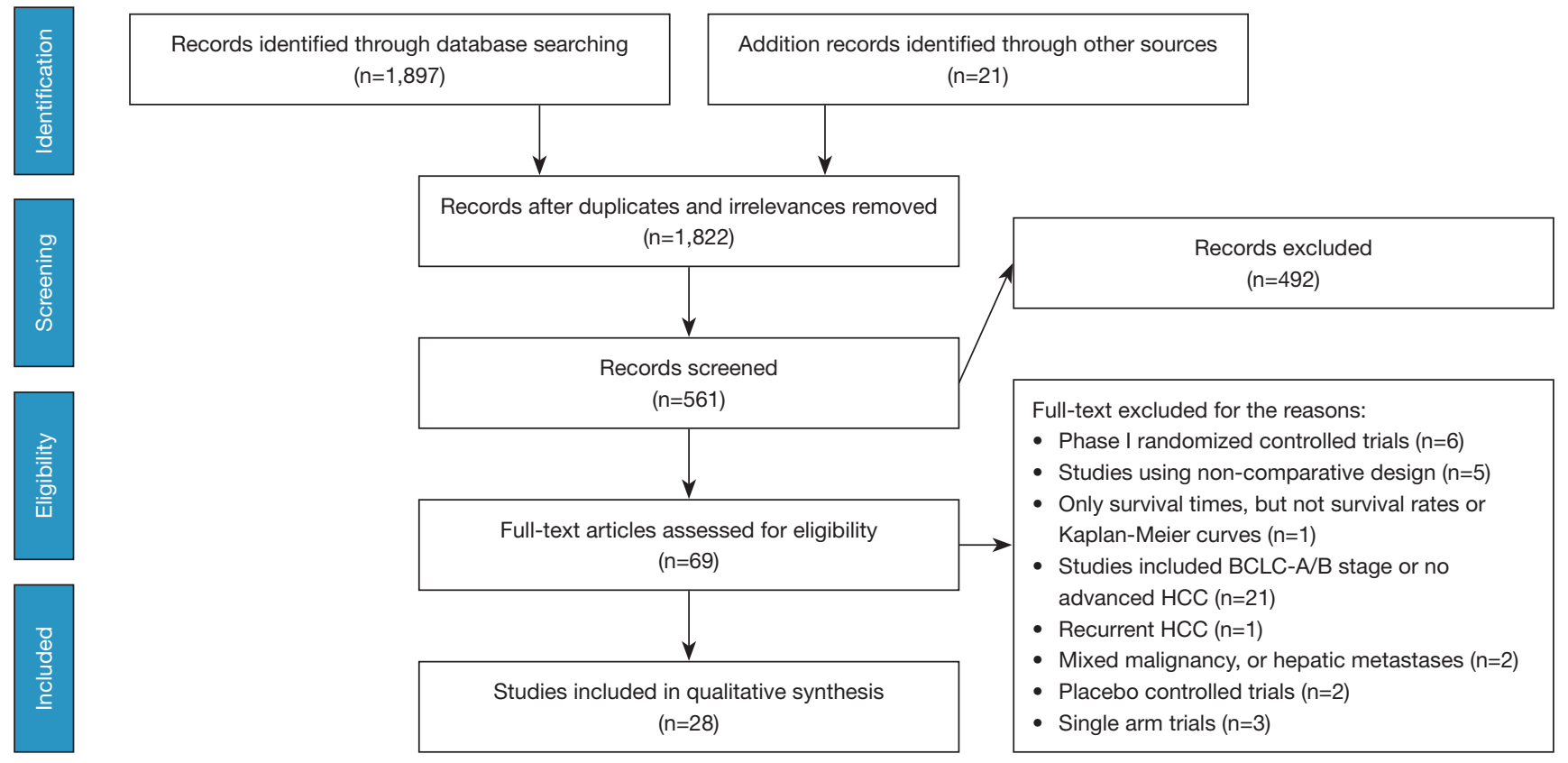

Figure 1 Flowchart of study inclusion. BCLC, Barcelona Clinic Liver Cancer; HCC, hepatocellular carcinoma.

1-year survival, and the effective rate (CR + PR, according to the RECIST). Overall study-level literature quality evaluation was presented in Table 2, according to the NOS, and the Cochrane risk of bias tool.

\section{Main results of efficacy of sequential therapy by direct meta-analysis}

Results from direct pair-wise meta-analysis of interventional therapies for advanced HCC are shown in Table 3. For 1 -year OS, there was no significant difference between HAIC plus sorafenib and sorafenib $(\mathrm{P}=0.356)$. Compared with the combination of TACE or RAF and sorafenib, sorafenib alone ( $\mathrm{P}=0.008$, OR: 2.25, 95\% CI: 1.235-4.009), $(\mathrm{P}=0.019$, OR: $2.890,95 \% \mathrm{CI}: 1.252-12.088)$ and TACE alone ( $\mathrm{P}=0.006$, OR: $2.488,95 \% \mathrm{CI}: 1.301-4.759) \mathrm{did}$ not benefit 1-year survival rate; but, when compared with the HAIC and sorafenib alone, HAIC was associated with higher 1-year survival rate for advanced HCC patients ( $\mathrm{P}=0.006$, OR: $4.778,95 \%$ CI: $1.555-14.680)$.

Similarly, compared with sorafenib alone, there was apparent increased ORR rate in patients who received HAIC alone ( $\mathrm{P}=0.013$, OR: 3.611, 95\% CI: 1.306-9.984) and TACE plus sorafenib $(\mathrm{P}<0.001$, OR: $4.502,95 \% \mathrm{CI}$ : 2.208-9.180). Meanwhile, compared with sorafenib or TACE alone, the combination of HAIC plus sorafenib
( $\mathrm{P}=0.155$, OR: 7.147, 95\% CI: 0.476-107.191) and TACE plus sorafenib ( $\mathrm{P}=0.110$, OR: 2.838, 95\% CI: 0.789-10.214) did not benefit ORR rate (Table 3).

For grade 3 or $4 \mathrm{AE}$, TACE alone was associated with lower AE than TACE plus sorafenib ( $\mathrm{P}=0.022$, OR: 1.467, 95\% CI: 1.057-2.062), and as compared with sorafenib alone, there was not any $\mathrm{AE}$ rates benefit in patients who received any combination therapy with sorafenib (Table 3).

\section{Main results of efficacy of sequential therapy by network meta-analysis}

The 28 studies covered the presently used 10 categories of main therapies including TACE, sorafenib, HAIC, TACE + sorafenib, TACE + RT, RFA + sorafenib, HAIC + sorafenib, $\mathrm{HAIC}+\mathrm{RT}, \mathrm{HAIC}+\mathrm{RFA}$, TACE + RT + sorafenib with a total of 3,675 participants. We conducted network metaanalysis to comparison of treatment efficacy and safety of all above relevant targeted options for advanced HCC, which included direct (head-to-head) and indirect treatment comparisons of OS, ORR and AEs, and Figure 2 shows the overall comparison network. Node-splitting models were conducted to assess the inconsistency by testing the difference between the direct and indirect effect. If the $\mathrm{P}$ value is more than 0.05 , it indicates that the difference between the direct and indirect effect was not significant (Table 4). 


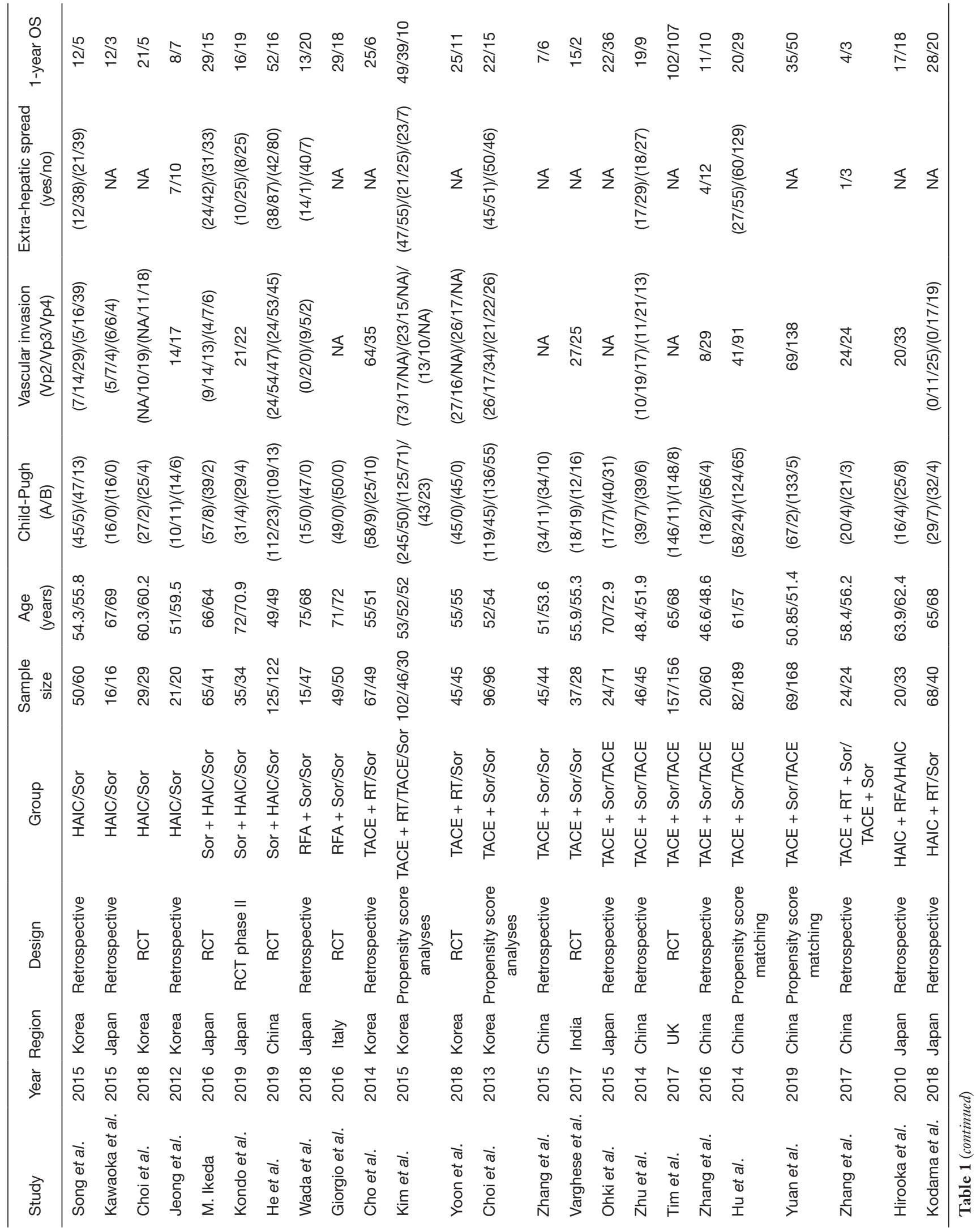




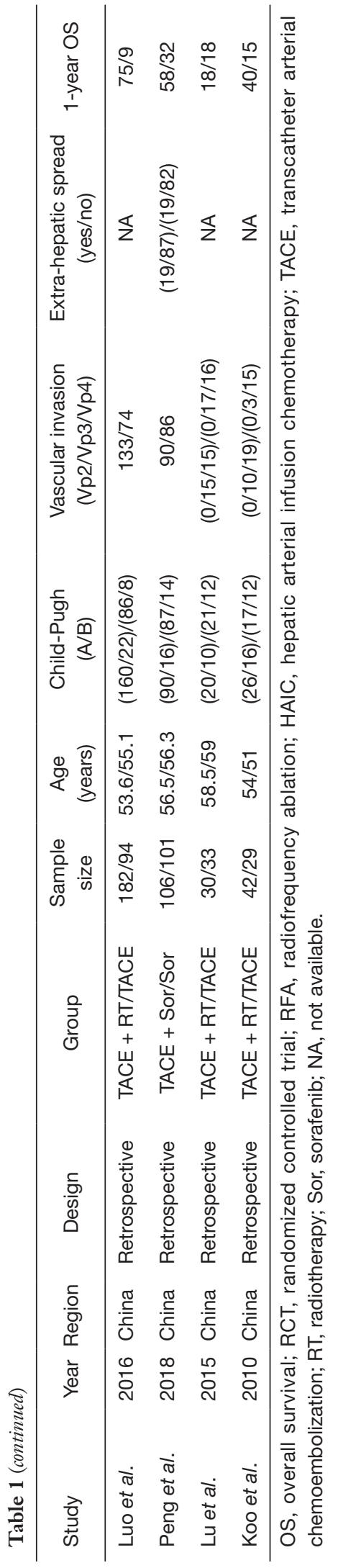

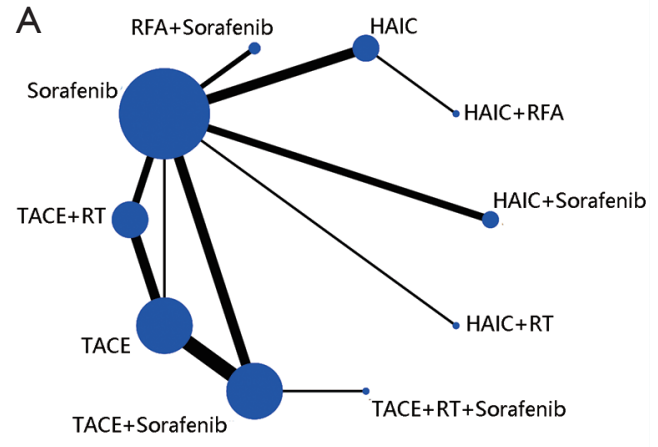
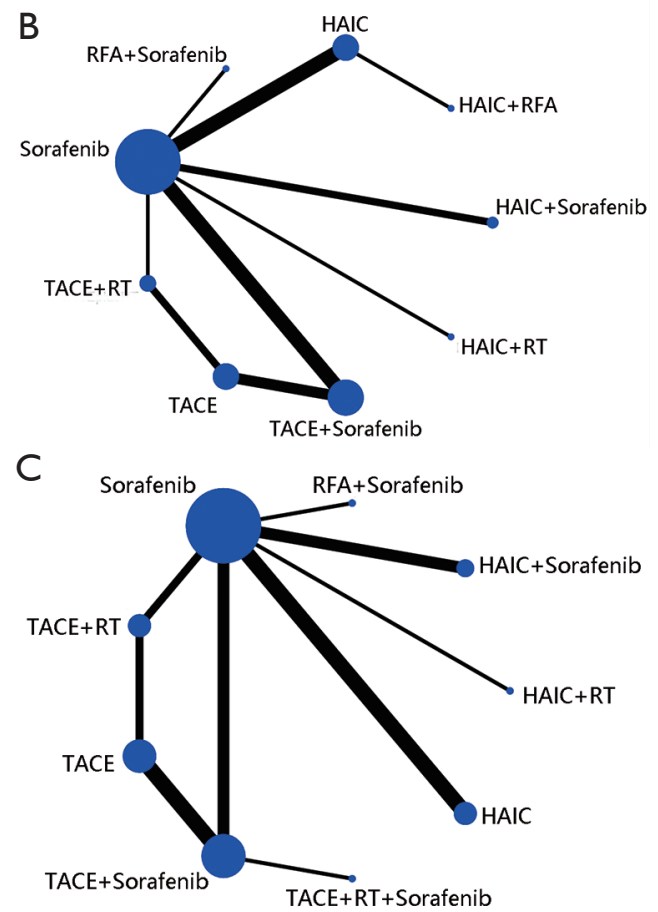

Figure 2 Network of included studies with the available direct comparisons. The size of the nodes is proportional to the number of patients (in parentheses) to receive the treatment. The width of the lines is proportional to the number of trials (beside the line) comparing the connected treatments. (A) For 1-year OS direct comparisons; (B) for ORR direct comparisons; (C) for AEs direct comparisons. OS, overall survival; ORR, overall response rate; $\mathrm{AE}$, adverse event; RFA, radiofrequency ablation; HAIC, hepatic arterial infusion chemotherapy; TACE, transcatheter arterial chemoembolization; RT, radiotherapy.

For OS, we used 1-year survival as an indicator and, Figure $2 A$ showed relative parametric data regarding 28 trials containing 3,675 patients reported. To analyze short-term efficacy ORR, 20 trials, including 9 treatment strategies or combinations, and sample-based relative parameter data 
Table 2 Quality assessment of included studies

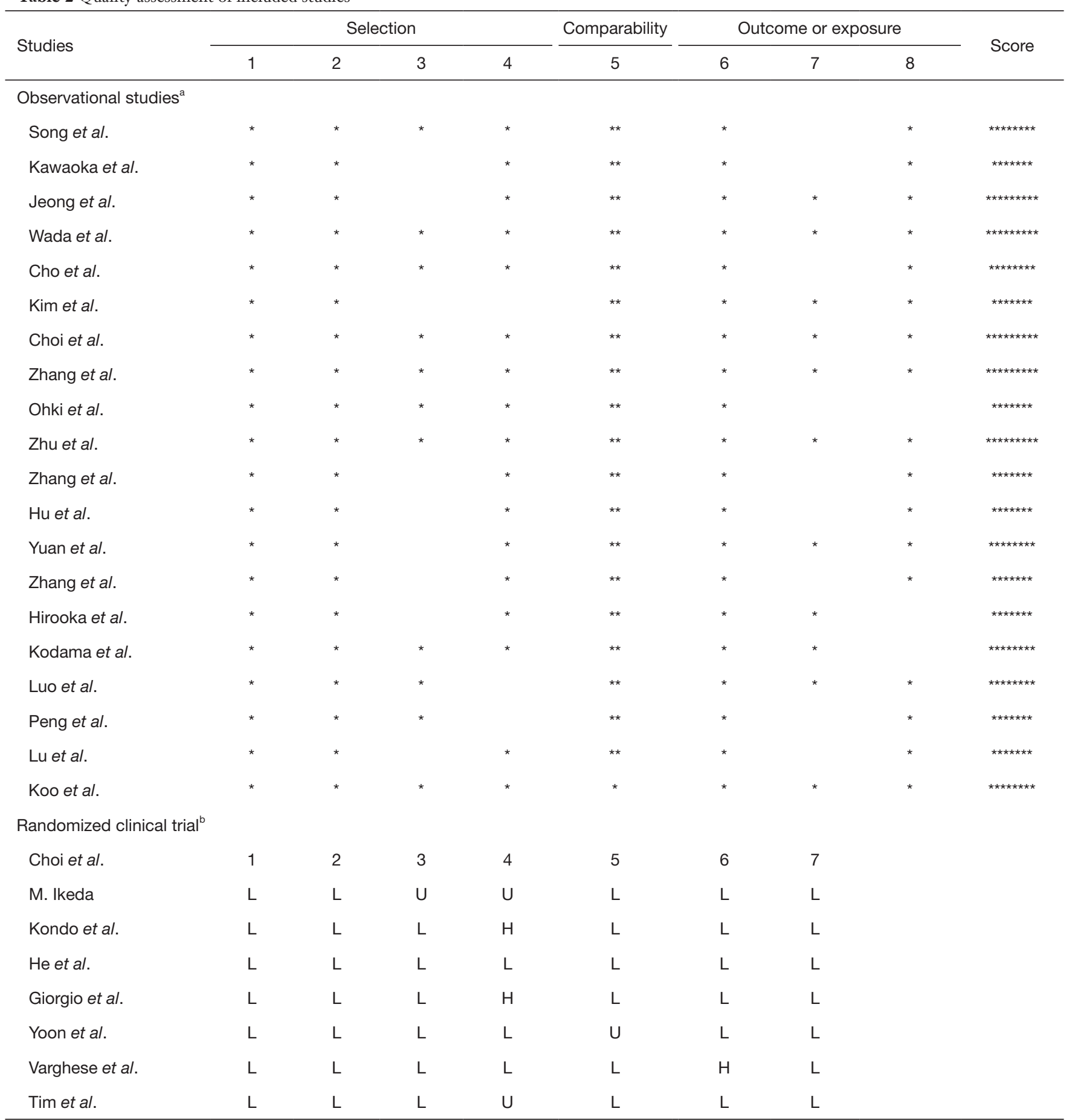

${ }^{a}$, the quality of the observational studies was performed using the NOS ( ${ }^{*}$ represents one star). ${ }^{b}$, the quality of the RCT was assessed by Cochrane risk of bias tool ( $\mathrm{L}=$ low risk, $\mathrm{H}$ = high risk, $\mathrm{U}=$ unclear risk). 1: random sequence generation; 2: allocation concealment; 3 : blinding of participants and researchers; 4: blinding of outcome assessment; 5: incomplete outcome data; 6: selective reporting; 7: other bias. NOS, Newcastle-Ottawa Quality Assessment Scale; RCT, randomized controlled trial. 
Table 3 Outcomes in the traditional meta-analysis

\begin{tabular}{|c|c|c|c|}
\hline Treatment method & Number of included studies & Results of pair-wise meta-analysis & $P$ value \\
\hline \multicolumn{4}{|l|}{ OS } \\
\hline HAIC vs. sorafenib & 4 & $4.778(1.555,14.680)$ & 0.006 \\
\hline HAIC + sorafenib vs. sorafenib & 3 & $1.707(0.537,5.404)$ & 0.365 \\
\hline RFA + sorafenib vs. sorafenib & 2 & $2.890(1.252,12.088)$ & 0.019 \\
\hline TACE + RT vs. sorafenib & 3 & $4.373(2.364,8.089)$ & $<0.001$ \\
\hline TACE + RT vs. TACE & 4 & $2.033(0.919,4.497)$ & 0.080 \\
\hline TACE + sorafenib vs. TACE & 6 & $2.488(1.301,4.759)$ & 0.006 \\
\hline \multicolumn{4}{|l|}{ ORR } \\
\hline TACE + sorafenib vs. sorafenib & 4 & $4.502(2.208,9.180)$ & $<0.001$ \\
\hline TACE + sorafenib vs. TACE & 3 & $2.838(0.789,10.214)$ & 0.110 \\
\hline TACE + RT vs. TACE & 2 & $2.193(0.997,4.823)$ & 0.051 \\
\hline \multicolumn{4}{|l|}{$\mathrm{AE}$} \\
\hline HAIC vs. sorafenib & 4 & $1.179(0.411,3.385)$ & 0.760 \\
\hline HAIC + sorafenib vs. sorafenib & 3 & $1.258(0.845,1.872)$ & 0.258 \\
\hline TACE + RT vs. sorafenib & 2 & $0.411(0.078,2.167)$ & 0.294 \\
\hline TACE + sorafenib vs. sorafenib & 3 & $1.309(0.862,1.986)$ & 0.207 \\
\hline
\end{tabular}

OS, overall survival; ORR, overall response rate; $A E$, adverse event; RFA, radiofrequency ablation; HAIC, hepatic arterial infusion chemotherapy; TACE, transcatheter arterial chemoembolization; RT, radiotherapy.

without previously targeted were reported (Figure 2B). For participants who have not received any treatment in the past, 20 trials, including 9 treatment strategies reported relevant raw data, and the results showed that patients HAIC + RT had the lowest adverse reaction rate followed by TACE + RT (Figure 2C).

After a comprehensive evaluation of the network metaanalysis results for different kinds of interventional therapies (Figure 3), we could observe that for patients with BCLC-C who have not received systemic treatment, HAIC combined with RAF has the highest probability of obtaining the best OS rate of 1 year (SUCRA, 0.95), which had ranked the first in hypothetical cases, followed by HAIC (SUCRA, 0.75) (Figure 3A). Based on the observed results, it was detected that HAIC plus RFA may have the best clinical efficacy in ORR for patients (SUCRA, 0.86) (Figure 3B); it was most probable that HAIC plus RFA was the best treatment followed by RFA plus sorafenib. On the other hand, combined RT has the greatest safety in reducing AEs, AEs occurring in patients who have received targeted drug sorafenib therapy (SUCRA, 0.51) (Figure 3C).

The ranking table in Figure 4 shows the comparison of the efficacy of all treatment regimens against each other in terms of OS, ORR and AEs in treating advanced HCC. Direct and indirect comparison of OS for patients with BCLC-C stage treated with all treatment regimens each other showed that for all treatment regimens, patients showed significant clinical benefit when compared with sorafenib (Figure 4A). For ORR of different interventional therapies, Figure $4 B$ shows HAIC plus RFA also was the 
Table 4 Assessment of inconsistency between direct and indirect evidence

\begin{tabular}{|c|c|c|c|c|}
\hline Treatment comparisons & Direct effect & Indirect effect & Overall & $P$ value of node-splitting method \\
\hline Sorafenib, TACE & $-0.89(-2.90,1.09)$ & $0.31(-0.61,1.22)$ & $0.11(-0.71,0.95)$ & 0.25 \\
\hline Sorafenib, TACE plus RT & $1.59(0.46,2.74)$ & $0.46(-0.96,1.81)$ & $1.12(0.25,1.99)$ & 0.19 \\
\hline Sorafenib, TACE plus sorafenib & $0.90(-0.06,1.87)$ & $1.26(-0.15,2.70)$ & $1.00(0.23,1.81)$ & 0.68 \\
\hline TACE, TACE plus sorafenib & $0.97(0.22,1.79)$ & $0.64(-0.93,2.19)$ & $0.89(0.26,1.58)$ & 0.68 \\
\hline \multicolumn{5}{|l|}{ ORR } \\
\hline Sorafenib, TACE plus RT & $2.33(-0.63,5.55)$ & $1.38(-1.63,4.26)$ & $1.81(-0.13,3.76)$ & 0.60 \\
\hline Sorafenib, TACE plus sorafenib & $1.68(0.29,3.18)$ & $2.68(-1.26,6.93)$ & $1.75(0.53,3.04)$ & 0.61 \\
\hline \multicolumn{5}{|l|}{$\mathrm{AE}$} \\
\hline Sorafenib, TACE plus RT & $-1.04(-2.06,-0.02)$ & $-0.01(-1.60,1.48)$ & $-0.74(-1.52,0.05)$ & 0.27 \\
\hline Sorafenib, TACE plus sorafenib & $0.29(-0.28,0.87)$ & $-0.77(-2.55,0.85)$ & $0.20(-0.37,0.73)$ & 0.22 \\
\hline TACE, TACE plus RT & $0.01(-0.99,1.35)$ & $-1.06(-2.22,0.25)$ & $0.43(-1.28,0.40)$ & 0.20 \\
\hline TACE, TACE plus sorafenib & $0.37(-0.05,0.83)$ & $1.27(-0.02,2.94)$ & $0.48(0.02,0.96)$ & 0.20 \\
\hline
\end{tabular}

OS, overall survival; ORR, overall response rate; AE, adverse event; HAIC, hepatic arterial infusion chemotherapy; TACE, transcatheter arterial chemoembolization; RT, radiotherapy.

best treatment option followed by RFA plus sorafenib. In the safety analysis, all included studies were retrieved grade 3 or 4 AEs under the condition that the authors reported AEs in different treatment strategies. Typically, the major differences were related to the well-known sorafenibassociated toxicities, including stomatitis, diarrhea, handfoot skin reaction, rash, and bleeding (Figure 4C).

\section{Discussion}

This network meta-analysis is based on 28 studies, including 3,675 participants, comparing the main treatments of BCLC-C patients, as well as the clinical benefits and AEs of different treatments. Our results indicate that HAIC plus RAF provides OS advantages and short-term effectiveness compared to other treatments, but may increase toxic effects. However, TACE plus sorafenib did not bring any benefit to the OS rate, and provided higher adverse reactions for this part of HCC patients.

Meta-analysis shows that HAIC plus sorafenib can improve the survival benefit of patients with BCLC-C HCC, and does not increase its adverse reactions. Compared with HAIC alone group, the HAIC combined with RFA group was associated with a higher survival rate, which could not be observed in the HAIC combined with sorafenib group and other combined therapy regimens. Moreover, compared with HAIC or TACE alone group, the combination of sorafenib did not significantly improve patient survival.

For advanced HCC patients who are not suitable for radical local regional therapy, such as resection, liver transplantation or local regional therapy, platinum-based systemic chemotherapy is still the preferred treatment (5). HAIC as a special systemic chemotherapy was widely used in Asia, especially in Japan. In addition to sorafenib, HAIC is also regarded as one of the treatment options according to the Japanese HCC management guideline for advanced HCC with macrovascular invasion (MVI) (VP3/4) (29). The regimens used in HAIC include CDDP, interferon (IFN), 5-FU alone, or their combination therapy takes different forms (29). Recently, more and more reports have reported that HAIC can improve tumor response rate and patient survival rate (42-44). As previously reported, several 


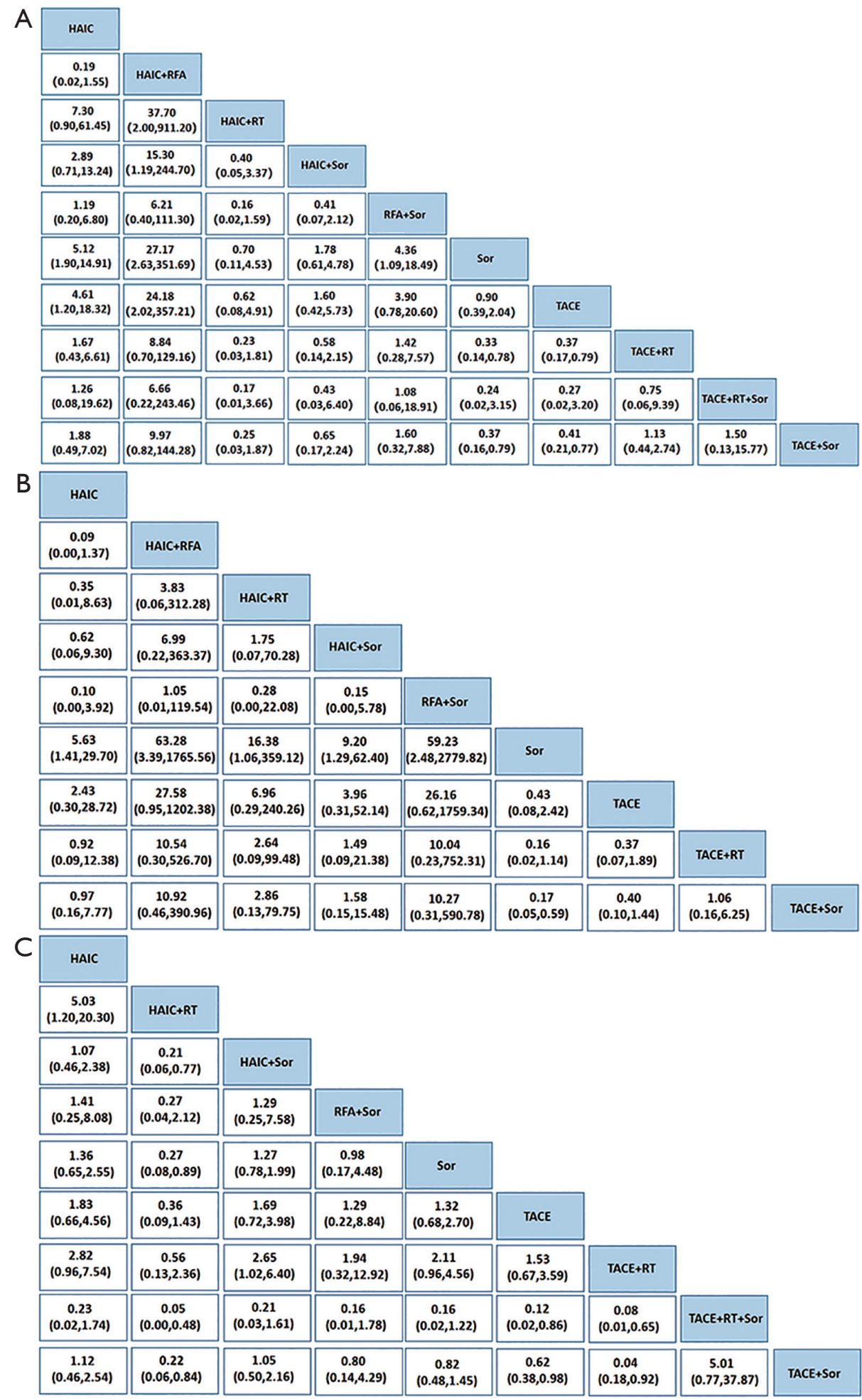

Figure 3 The network meta-analysis results for different kinds of interventional therapies. (A) One-year OS of different kinds of interventional therapies; (B) ORR of different kinds of interventional therapies; (C) AEs of different kinds of interventional therapies. OS, overall survival; ORR, overall response rate; AE, adverse event; RFA, radiofrequency ablation; HAIC, hepatic arterial infusion chemotherapy; TACE, transcatheter arterial chemoembolization; RT, radiotherapy; Sor, sorafenib. 


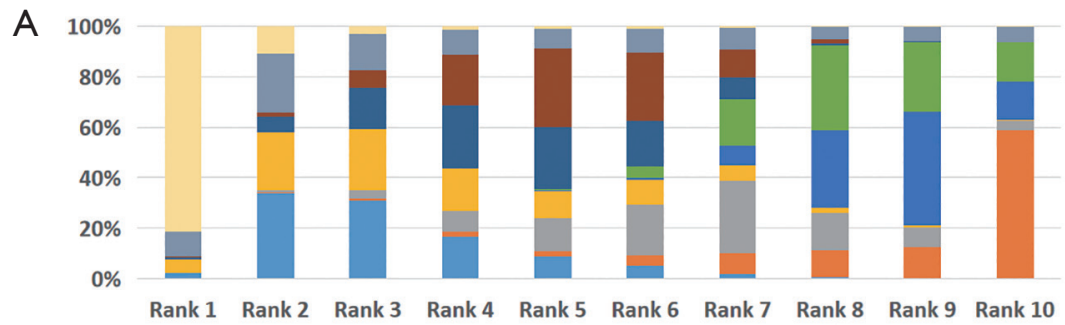

B
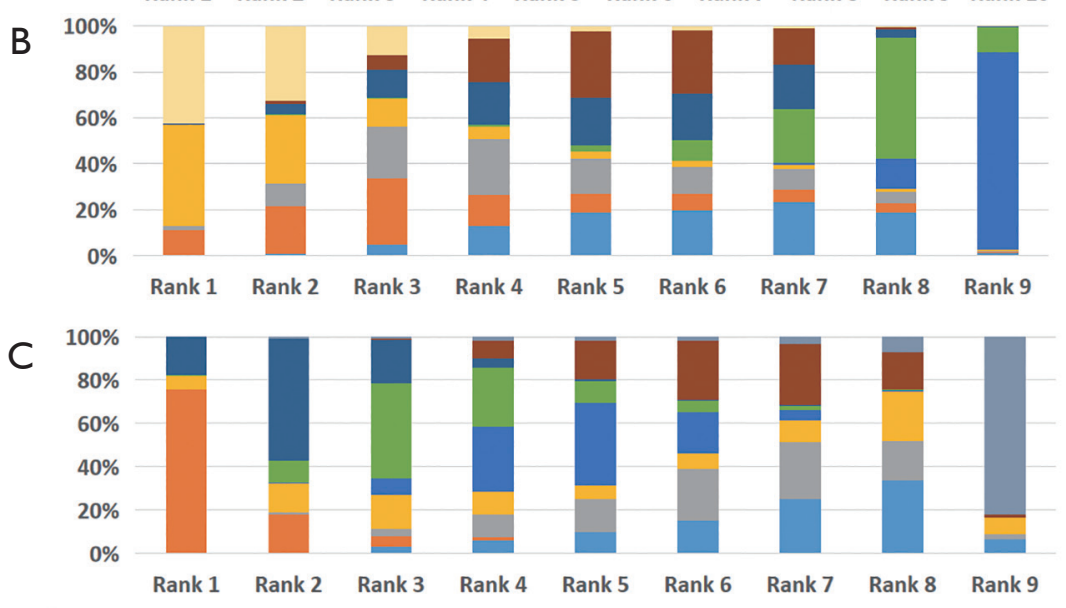

\begin{tabular}{lccc}
\hline \multirow{2}{*}{ Treatment method } & \multicolumn{3}{c}{ SCURA, \% } \\
\cline { 2 - 4 } & OS & ORR & AE \\
\hline HAIC & 75.3 & 37.3 & 27.6 \\
HAIC + RT & 12 & 68.1 & 95.7 \\
HAIC + sorafenib & 38.6 & 57.7 & 35.1 \\
RFA + sorafenib & 69.1 & 85.4 & 47.7 \\
Sorafenib & 14.8 & 1.9 & 51.8 \\
TACE & 19.1 & 18 & 7.4 \\
TACE + RT & 59.9 & 48.4 & 85.0 \\
TACE + sorafenib & 54.1 & 46.5 & 33.5 \\
TACE + RT + sorafenib & 61.3 & - & 6.2 \\
HAIC + RFA & 95.7 & 86.7 & - \\
\hline
\end{tabular}

Figure 4 Results of rank test for different interventional therapies. (A) For 1-year OS of different interventional therapies; (B) for ORR of different interventional therapies; (C) for AEs of different interventional therapies. OS, overall survival; ORR, overall response rate; AE, adverse event; RFA, radiofrequency ablation; HAIC, hepatic arterial infusion chemotherapy; TACE, transcatheter arterial chemoembolization; RT, radiotherapy; SUCRA, surface under the cumulative ranking.

studies have shown survival benefits of HAIC for advanced HCC without extrahepatic metastasis (EHM) (45), with response rates ranging from $20.8 \%$ to $52 \%(43,46-49)$. Studies also have revealed that sequential HAIC after liver resection for advanced HCC with portal vein tumor thrombosis (PVTT) is also extremely effective $(47,50)$.

TACE and HAIC treatment are mainly based on the differences in the population. TACE treatment is mainly targeted at patients with intermediate stage HCC, specifically (51). While patients with advanced HCC targeted by HAIC, especially those with advanced HCC with heavy burden of intrahepatic lesions. HAIC have more advantages compared with sorafenib in previous clinical studies. At present, many hospitals are exploring the HAIC (FOLFOX) contrast with sorafenib III prospective head-tohead randomized controlled clinical trials in advanced HCC.

Advanced HCC is often accompanied by PVTT. Sorafenib, an oral multi-kinase inhibitor that blocks tumor cell proliferation and angiogenesis, significantly improved OS and has become the first-line option for BCLC stage C, which including cases complicated with MVI and EHM $(52,53)$.
However, the use of sorafenib has gradually been limited because its severity and frequency AEs, ungratified efficacy application and high cost $(51,54)$. TACE in combination with sorafenib seems to be the option for advanced HCC, but its high cost and intolerable adverse reactions has resulted in required dose reductions or interruptions in sorafenib-treated patients (55). Moreover, patients in the sorafenib group discontinued TACE treatments earlier (56,57). These are the reasons why TACE combined with sorafenib does not show a difference in survival.

Conversely, RFA, less invasive than hepatectomy, can be manipulated easily and can achieve complete coagulative necrosis. So, many liver cancer treatment guidelines consider RFA as effective as surgical resection to treat small HCC $<3 \mathrm{~cm}$ in diameter (57). Some studies indicate that RFA as a complementary treatment plays a very important role in the treatment of advanced HCC. With the developments of technique for RFA, large HCC and PVTT also can be treated by RFA (58). HAIC can increase the concentrate of drug within the tumor and reduce the waste of peripheral drugs, which helps reduce systemic adverse 
reactions of patients. Continuous infusion chemotherapy using the implanted drug delivery systems to deliver drugs regularly through the appropriate hepatic arteries may be an effective way to control tumor progression and prolong survival (58). More importantly, it has effective anti-tumor activity and reduces systemic adverse reactions. At the same time, RFA can synergistically increase tumor local necrosis. So, RFA is an effective tumor reduction option before HAIC treatment.

This network meta-analysis suggests that combination therapy with HAIC and RFA did not worsen liver function and had a best survival benefit for advanced HCC patients compared with other sequential therapy. However, there were still some limitations existed in this study. First, current systemic therapy of HCC consists of receptor TKIs and checkpoint inhibitors. A timely consideration of systemic therapy is an important part of advanced HCC management. With a lack of a direct comparison between CIT strategies and observation alone for patients with advanced HCC, the question of which CIT combined with local therapy strategies is optimal for the patient still remains inconclusive. Second, the baseline characteristics of all the enrolled studies might affect the possible heterogeneity as well as the final results, such as the dose of sorafenib and HAIC, the duration and frequency of medication, the type of tumor and portal vein thrombus, and the primary tumor size, and there were slightly differences between the studies and may be confounding factor. Thirdly, the small sample size of some studies may lead to an overestimate of the results. Consequently, a multidisciplinary management and close collaboration between hepatology, oncology, and interventional radiology centered over patients' goals for therapy is essential for optimal outcomes and multicenter and high-quality RCTs with large sample sizes are expecting to confirm the advantages of the combined therapy for advanced HCC in the future.

\section{Conclusions}

In conclusion, HAIC may be a valuable therapeutic strategy for advanced HCC patients to prevent recurrence and metastasis after RFA, as well as in improving patient prognosis and quality of life. Addition, in this meta-analysis, there was not directly compared to TACE-sorafenib and HAIC-RAF, but it was suggested that HAIC-RAF appeared to be better to TACE plus sorafenib in advancedstage HCC. So, we also speculate that the main difference between TACE and HAIC is the adaptation to the population. TACE is mainly for patients with intermediatestage HCC, while HAIC is for patients with advanced HCC, especially advanced HCC with heavy intrahepatic lesions. TACE combined with sorafenib may contribute to improving survival prognosis of intermediate-stage HCC.

\section{Acknowledgments}

We gratefully appreciate the efforts and contributions of doctors, nurses, and technical staff at Tangdu Hospital, Air Force Military Medical University.

Funding: None.

\section{Footnote}

Reporting Checklist: The authors have completed the PRISMA reporting checklist. Available at https://tgh. amegroups.com/article/view/10.21037/tgh-20-318/rc

Conflicts of Interest: All authors have completed the ICMJE uniform disclosure form (available at https://tgh.amegroups. com/article/view/10.21037/tgh-20-318/coif). The authors have no conflicts of interest to declare.

Ethical Statement: The authors are accountable for all aspects of the work in ensuring that questions related to the accuracy or integrity of any part of the work are appropriately investigated and resolved.

Open Access Statement: This is an Open Access article distributed in accordance with the Creative Commons Attribution-NonCommercial-NoDerivs 4.0 International License (CC BY-NC-ND 4.0), which permits the noncommercial replication and distribution of the article with the strict proviso that no changes or edits are made and the original work is properly cited (including links to both the formal publication through the relevant DOI and the license). See: https://creativecommons.org/licenses/by-nc-nd/4.0/.

\section{References}

1. Feng RM, Zong YN, Cao SM, et al. Current cancer situation in China: good or bad news from the 2018 Global Cancer Statistics? Cancer Commun (Lond) 2019;39:22.

2. Bray F, Ferlay J, Soerjomataram I, et al. Global cancer statistics 2018: GLOBOCAN estimates of incidence and mortality worldwide for 36 cancers in 185 countries. CA 
Cancer J Clin 2018;68:394-424.

3. Bangaru S, Marrero JA, Singal AG. Review article: new therapeutic interventions for advanced hepatocellular carcinoma. Aliment Pharmacol Ther 2020;51:78-89.

4. Fujino H, Kimura T, Aikata H, et al. Role of 3-D conformal radiotherapy for major portal vein tumor thrombosis combined with hepatic arterial infusion chemotherapy for advanced hepatocellular carcinoma. Hepatol Res 2015;45:607-17.

5. Doycheva I, Thuluvath PJ. Systemic therapy for advanced hepatocellular carcinoma: an update of a rapidly evolving field. J Clin Exp Hepatol 2019;9:588-96.

6. Meyer T, Fox R, Ma YT, et al. Sorafenib in combination with transarterial chemoembolisation in patients with unresectable hepatocellular carcinoma (TACE 2): a randomised placebo-controlled, double-blind, phase 3 trial. Lancet Gastroenterol Hepatol 2017;2:565-75.

7. Ding M, Wang Y, Chi J, et al. Is adjuvant cellular immunotherapy essential after TACE-predominant minimally-invasive treatment for hepatocellular carcinoma? A systematic meta-analysis of studies including 1774 patients. PLoS One 2016;11:e0168798.

8. Lee MP, Ravenel JD, Hu RJ, et al. Targeted disruption of the Kvlqt1 gene causes deafness and gastric hyperplasia in mice. J Clin Invest 2000;106:1447-55.

9. Choi JH, Chung WJ, Bae SH, et al. Randomized, prospective, comparative study on the effects and safety of sorafenib vs. hepatic arterial infusion chemotherapy in patients with advanced hepatocellular carcinoma with portal vein tumor thrombosis. Cancer Chemother Pharmacol 2018;82:469-78.

10. He M, Li Q, Zou R, et al. Sorafenib plus hepatic arterial infusion of oxaliplatin, fluorouracil, and leucovorin vs sorafenib alone for hepatocellular carcinoma with portal vein invasion: a randomized clinical trial. JAMA Oncol 2019;5:953-60.

11. Kondo M, Morimoto M, Kobayashi S, et al. Randomized, phase II trial of sequential hepatic arterial infusion chemotherapy and sorafenib versus sorafenib alone as initial therapy for advanced hepatocellular carcinoma: SCOOP-2 trial. BMC Cancer 2019;19:954.

12. Giorgio A, Merola MG, Montesarchio L, et al. Sorafenib combined with radio-frequency ablation compared with sorafenib alone in treatment of hepatocellular carcinoma invading portal vein: a western randomized controlled trial. Anticancer Res 2016;36:6179-83.

13. Ikeda M, Shimizu S, Sato T, et al. Sorafenib plus hepatic arterial infusion chemotherapy with cisplatin versus sorafenib for advanced hepatocellular carcinoma: randomized phase II trial. Ann Oncol 2016;27:2090-6.

14. Yoon SM, Ryoo BY, Lee SJ, et al. Efficacy and safety of transarterial chemoembolization plus external beam radiotherapy vs sorafenib in hepatocellular carcinoma with macroscopic vascular invasion: a randomized clinical trial. JAMA Oncol 2018;4:661-9.

15. Varghese J, Kedarisetty C, Venkataraman J, et al. Combination of TACE and sorafenib improves outcomes in BCLC stages B/C of hepatocellular carcinoma: a single centre experience. Ann Hepatol 2017;16:247-54.

16. Cheng AL, Thongprasert S, Lim HY, et al. Randomized, open-label phase 2 study comparing frontline dovitinib versus sorafenib in patients with advanced hepatocellular carcinoma. Hepatology 2016;64:774-84.

17. Chow PKH, Gandhi M, Tan SB, et al. SIRveNIB: Selective internal radiation therapy versus sorafenib in Asia-Pacific patients with hepatocellular carcinoma. J Clin Oncol 2018;36:1913-21.

18. Gandhi M, Choo SP, Thng CH, et al. Single administration of Selective Internal Radiation Therapy versus continuous treatment with sorafeNIB in locally advanced hepatocellular carcinoma (SIRveNIB): study protocol for a phase iii randomized controlled trial. BMC Cancer 2016;16:856.

19. Kudo M, Ueshima K, Yokosuka O, et al. Sorafenib plus low-dose cisplatin and fluorouracil hepatic arterial infusion chemotherapy versus sorafenib alone in patients with advanced hepatocellular carcinoma (SILIUS): a randomised, open label, phase 3 trial. Lancet Gastroenterol Hepatol 2018;3:424-32.

20. Vilgrain V, Pereira H, Assenat E, et al. Efficacy and safety of selective internal radiotherapy with yttrium-90 resin microspheres compared with sorafenib in locally advanced and inoperable hepatocellular carcinoma (SARAH): an open-label randomised controlled phase 3 trial. Lancet Oncol 2017;18:1624-36.

21. Yang Y, Lu Y, Wang C, et al. Cryotherapy is associated with improved clinical outcomes of Sorafenib therapy for advanced hepatocellular carcinoma. Cell Biochem Biophys 2012;63:159-69.

22. Cheng AL, Kang YK, He AR, et al. Safety and efficacy of tigatuzumab plus sorafenib as first-line therapy in subjects with advanced hepatocellular carcinoma: a phase 2 randomized study. J Hepatol 2015;63:896-904.

23. Cainap C, Qin S, Huang WT, et al. Linifanib versus Sorafenib in patients with advanced hepatocellular carcinoma: results of a randomized phase III trial. J Clin 
Oncol 2015;33:172-9.

24. Choi GH, Shim JH, Kim MJ, et al. Sorafenib alone versus sorafenib combined with transarterial chemoembolization for advanced-stage hepatocellular carcinoma: results of propensity score analyses. Radiology 2013;269:603-11.

25. Hu H, Duan Z, Long X, et al. Sorafenib combined with transarterial chemoembolization versus transarterial chemoembolization alone for advanced-stage hepatocellular carcinoma: a propensity score matching study. PLoS One 2014;9:e96620.

26. Jeong SW, Jang JY, Lee JE, et al. The efficacy of hepatic arterial infusion chemotherapy as an alternative to sorafenib in advanced hepatocellular carcinoma. Asia Pac J Clin Oncol 2012;8:164-71.

27. Kawaoka T, Aikata H, Hyogo H, et al. Comparison of hepatic arterial infusion chemotherapy versus sorafenib monotherapy in patients with advanced hepatocellular carcinoma. J Dig Dis 2015;16:505-12.

28. Kim GA, Shim JH, Yoon SM, et al. Comparison of chemoembolization with and without radiation therapy and sorafenib for advanced hepatocellular carcinoma with portal vein tumor thrombosis: a propensity score analysis. J Vasc Interv Radiol 2015;26:320-9.e6.

29. Kodama K, Kawaoka T, Aikata H, et al. Comparison of outcome of hepatic arterial infusion chemotherapy combined with radiotherapy and sorafenib for advanced hepatocellular carcinoma patients with major portal vein tumor thrombosis. Oncology 2018;94:215-22.

30. Luo JJ, Zhang ZH, Liu QX, et al. Endovascular brachytherapy combined with stent placement and TACE for treatment of HCC with main portal vein tumor thrombus. Hepatol Int 2016;10:185-95.

31. Ni JY, Sun HL, Luo JH, et al. Transarterial chemoembolization and sorafenib combined with microwave ablation for advanced primary hepatocellular carcinoma: a preliminary investigation of safety and efficacy. Cancer Manag Res 2019;11:9939-50.

32. Ohki T, Sato K, Yamagami M, et al. Efficacy of transcatheter arterial chemoembolization followed by sorafenib for intermediate/advanced hepatocellular carcinoma in patients in Japan: a retrospective analysis. Clin Drug Investig 2015;35:751-9.

33. Peng $Z$, Chen $S$, Wei $M$, et al. Advanced recurrent hepatocellular carcinoma: treatment with sorafenib alone or in combination with transarterial chemoembolization and radiofrequency ablation. Radiology 2018;287:705-14.

34. Wada Y, Takami Y, Matsushima H, et al. The safety and efficacy of combination therapy of sorafenib and radiotherapy for advanced hepatocellular carcinoma: a retrospective study. Intern Med 2018;57:1345-53.

35. Wan X, Zhai X, Yan Z, et al. Retrospective analysis of transarterial chemoembolization and sorafenib in Chinese patients with unresectable and recurrent hepatocellular carcinoma. Oncotarget 2016;7:83806-16.

36. Yuan J, Yin X, Tang B, et al. Transarterial chemoembolization (TACE) combined with sorafenib in treatment of HBV background hepatocellular carcinoma with portal vein tumor thrombus: a propensity score matching study. Biomed Res Int 2019;2019:2141859.

37. Zhang Y, Fan W, Wang Y, et al. Sorafenib with and without transarterial chemoembolization for advanced hepatocellular carcinoma with main portal vein tumor thrombosis: a retrospective Analysis. Oncologist 2015;20:1417-24.

38. Zhang YF, Wei W, Wang JH, et al. Transarterial chemoembolization combined with sorafenib for the treatment of hepatocellular carcinoma with hepatic vein tumor thrombus. Onco Targets Ther 2016;9:4239-46.

39. Zhang ZH, Liu QX, Zhang W, et al. Combined endovascular brachytherapy, sorafenib, and transarterial chemobolization therapy for hepatocellular carcinoma patients with portal vein tumor thrombus. World J Gastroenterol 2017;23:7735-45.

40. Zhou Z, Qin H, Weng L, et al. Clinical efficacy of DCCIK combined with sorafenib in the treatment of advanced hepatocellular carcinoma. J BUON 2019;24:615-21.

41. Zhu K, Chen J, Lai L, et al. Hepatocellular carcinoma with portal vein tumor thrombus: treatment with transarterial chemoembolization combined with sorafenib-a retrospective controlled study. Radiology 2014;272:284-93.

42. Ishida K, Hirooka M, Hiraoka A, et al. Treatment of hepatocellular carcinoma using arterial chemoembolization with degradable starch microspheres and continuous arterial infusion of 5 -fluorouracil. Jpn J Clin Oncol 2008;38:596-603.

43. Obi S, Yoshida H, Toune R, et al. Combination therapy of intraarterial 5-fluorouracil and systemic interferon-alpha for advanced hepatocellular carcinoma with portal venous invasion. Cancer 2006;106:1990-7.

44. Sakon M, Nagano H, Dono K, et al. Combined intraarterial 5 -fluorouracil and subcutaneous interferonalpha therapy for advanced hepatocellular carcinoma with tumor thrombi in the major portal branches. Cancer 2002;94:435-42.

45. Splawski I, Shen J, Timothy KW, et al. Genomic structure 
of three long QT syndrome genes: KVLQT1, HERG, and KCNE1. Genomics 1998;51:86-97.

46. Ando E, Tanaka M, Yamashita F, et al. Hepatic arterial infusion chemotherapy for advanced hepatocellular carcinoma with portal vein tumor thrombosis: analysis of 48 cases. Cancer 2002;95:588-95.

47. Miyaki D, Aikata H, Honda Y, et al. Hepatic arterial infusion chemotherapy for advanced hepatocellular carcinoma according to Child-Pugh classification. J Gastroenterol Hepatol 2012;27:1850-7.

48. Uka K, Aikata H, Takaki S, et al. Similar effects of recombinant interferon-alpha-2b and natural interferonalpha when combined with intra-arterial 5-fluorouracil for the treatment of advanced hepatocellular carcinoma. Liver Int 2007;27:1209-16.

49. Uka K, Aikata H, Takaki S, et al. Pretreatment predictor of response, time to progression, and survival to intraarterial 5 -fluorouracil/interferon combination therapy in patients with advanced hepatocellular carcinoma. J Gastroenterol 2007;42:845-53.

50. Fukuda S, Okuda K, Imamura M, et al. Surgical resection combined with chemotherapy for advanced hepatocellular carcinoma with tumor thrombus: report of 19 cases. Surgery 2002;131:300-10.

51. Chen QF, Wu PH, Huang T, et al. Efficacy of treatment regimens for advanced hepatocellular carcinoma: a network meta-analysis of randomized controlled trials. Medicine (Baltimore) 2019;98:e17460.

doi: $10.21037 / \operatorname{tgh}-20-318$

Cite this article as: Yang Z, Tong Y, Yang L, He X, Bao G, $\mathrm{Du} \mathrm{X}$. Identifying optimal therapies in patients with advanced hepatocellular carcinoma: a systematic review and network meta-analysis. Transl Gastroenterol Hepatol 2022;7:38.
52. Cheng AL, Kang YK, Chen Z, et al. Efficacy and safety of sorafenib in patients in the Asia-Pacific region with advanced hepatocellular carcinoma: a phase III randomised, double-blind, placebo-controlled trial. Lancet Oncol 2009;10:25-34.

53. Llovet JM, Ricci S, Mazzaferro V, et al. Sorafenib in advanced hepatocellular carcinoma. N Engl J Med 2008;359:378-90.

54. Pinter M, Hucke F, Graziadei I, et al. Advanced-stage hepatocellular carcinoma: transarterial chemoembolization versus sorafenib. Radiology 2012;263:590-9.

55. Kudo M, Imanaka K, Chida N, et al. Phase III study of sorafenib after transarterial chemoembolisation in Japanese and Korean patients with unresectable hepatocellular carcinoma. Eur J Cancer 2011;47:2117-27.

56. Lencioni R, Llovet JM, Han G, et al. Sorafenib or placebo plus TACE with doxorubicin-eluting beads for intermediate stage HCC: the SPACE trial. J Hepatol 2016;64:1090-8.

57. Yuan H, Lan Y, Li X, et al. Large hepatocellular carcinoma with local remnants after transarterial chemoembolization: treatment by sorafenib combined with radiofrequency ablation or sorafenib alone. Am J Cancer Res 2019;9:791-9.

58. Hirooka M, Koizumi Y, Kisaka Y, et al. Mass reduction by radiofrequency ablation before hepatic arterial infusion chemotherapy improved prognosis for patients with huge hepatocellular carcinoma and portal vein thrombus. AJR Am J Roentgenol 2010;194:W221-6. 